\title{
RE-PENSANDO LA COLONIALIDAD Y DECOLONIALIDAD EN EL ESPACIO DE LA EDUCACIÓN NO FORMAL DESDE LA INTERCULTURALIDAD CRÍTICA EN San Cristóbal de las Casas, Chiapas, México
}

\author{
RE-THINKING COLONIALITY AND DECONALIALITY FROM \\ A CRITICAL INTERCULTURAL PERSPECTIVE: THE CASE OF \\ A NON-FORMAL EDUCATION SPACE IN \\ San Cristobal de Las Casas, Chiapas, Mexico
}

\section{Arantzazu Giner Espín \\ arginer@ecosur.edu.mx}

Currículo: maestra en Ciencias y Desarrollo Rural, y maestra en Mediación Intercultural. Educadora social en espacios de educación no formal con niños, niñas, adolescentes y adultos y adultas. Sus líneas de investigación versan sobre la educación no formal y transformación social, interculturalidad crítica y pedagogía decolonial, género y pedagogía del sujeto.

\section{Antonio Saldívar Moreno \\ asaldivar@ecosur.mx}

Currículo: doctor en Comunicación, Cultura y Educación. Profesor-investigador en El Colegio de la Frontera Sur. Miembro del Sistema Nacional de Investigadores en México. Sus líneas de investigación son educación intercultural, sistematización y evaluación de procesos educativos, participación social y desarrollo comunitario, estudios ambientales y educación en valores.

\section{José María Duarte Cruz}

jduarte@ecosur.mx

Currículo: doctor en Filosofía con orientación en Trabajo Social y Políticas Comparadas de Bienestar Social. Catedrático Conacyt, comisionado en la Universidad Autónoma Metropolitana, Unidad Xochimilco. Sus líneas de investigación abordan las dimensiones del acoso escolar, prevención de la violencia familiar, género y educación, y estudios de educación para la paz.

\section{Charles Keck}

charliekeck@gmail.com

Currículo: doctor en Educación. Académico adscrito al Departamento de Sociedad y Cultura de El Colegio de la Frontera Sur. Miembro del Sistema Nacional de Investigadores en México. Sus líneas de investigación son la experiencia laboral docente, la formación ética-socioemocional del docente, la ética del cuidado de sí, la innovación y procesos de transformación educativa.

Recibido: 31 de agosto de 2017. Aceptado para su publicación: 16 de marzo de 2018. Recuperado de: https://sinectica.iteso.mx/index.php/SINECTICA/article/view/794 DOI: 10.31391/S2007-7033(2018)0050-005

\section{Resumen}

Los espacios de educación no formal en Chiapas proliferaron en su mayoría después del movimiento zapatista de 1994, y algunas de sus actividades han sido cuestionadas por presentar contradicciones en su lucha contra la exclusión social. En este artículo, con una actitud reflexiva y crítica, profundizamos desde un espacio de educación no formal entre lo que se promueve como transformación social del problema de la exclusión y la contradicción sutil en determinados discursos y acciones. 
Retomamos la interculturalidad crítica para re-pensar el espacio, las relaciones y la práctica socioeducativa que se dan en Amalgama, $\mathrm{AC}$, que, con un nombre ficticio, representa un espacio de educación no formal en San Cristóbal de Las Casas, Chiapas, México. Por un lado, visibilizamos elementos de la estructura colonial-capitalista-patriarcal, concretamente de la colonialidad, que aparecen en el espacio, las relaciones y la práctica socioeducativa de esa asociación y, por otro, recuperamos algunas subjetividades de las personas-sujetos educadoras que resignifican estos espacios, relaciones y prácticas para, así, reflexionar sobre transformaciones sociales potenciadas desde la pedagogía decolonial.

Palabras clave: espacio de educación no formal, interculturalidad crítica, práctica socioeducativa, colonialidad, (des)situaciones, aproximación decolonial.

\begin{abstract}
In Chiapas, a Mexican southern state, non-formal education spaces have increased rapidly after "Zapatista Movement" in 1994. Nevertheless, recently some scholars have questioned certain activities carried out in these spaces because they find contradictions in the struggle against social exclusion. In this research paper, we analyzed with a reflexive point of view and with a critical approach the results obtained in a study performed in one of these spaces of non-formal education in which social transformation is promoted as an answer for the social exclusion problem. Here, we pointed out the delicate line that exists between actions and discourses in these non-formal education spaces. To reach this goal, we worked with a critical intercultural perspective and thus, we carried out an exercise to re-thinking space, relationships and social-educative practices in Amalgama, A. C. (fictional name), a non-formal education space located in San Cristobal de las Casas, Chiapas. As a result, we found out, on one hand that in the space, in the relationships and in the social-educative practice of Amalgama, A.C. there are elements of the colonialcapitalist-patriarchal structure, and such elements are closer to the coloniality than we expected at the beginning. On the other hand, this exercise allowed us to recover the perceptions of the educators to re-signifying this space, relationships and social-educative practice to eventually make social transformations encouraged them since the decolonial pedagogical approach.
\end{abstract}

Keywords: non formal education espace, critical intercultural, social-educative practice, coloniality, (des)situation, decolonial approach.

\title{
ESPACIOS DE EDUCACIÓN NO FORMAL Y EL MOVIMIENTO ZAPATISTA EN SAN CRISTÓBAL de LAS CASAS, ChIAPAS, MÉxico
}

os espacios de educación no formal en esta investigación son aquellos que trabajan de manera preferente con grupos en situación de riesgo, exclusión, violencia, vulnerabilidad y pobreza, y se consolidan como asociaciones civiles que no pertenecen a un sistema oficial estatal certificado, como la escuela (Trilla, 1993); son espacios que se crean por motivación propia de una o varias personas y desarrollan un tipo de educación diferente a la educación formal en espacio, metodología y contenido (Smitter, 2006; Pincheira, 2013). Esta diferencia sucede porque las asociaciones civiles de educación no formal se desarrollan para cumplir con una visón, misión y objetivo que, en muchas ocasiones, tienen que ver con aminorar la exclusión social de la población con la que trabajan y no se consolidan para cumplir únicamente con el ejercicio de la educación básica oficial, aunque en 
sus programas puedan incluir, de modo extraescolar, algún tipo de apoyo educativo. Por ello, en esta investigación hablamos de una asociación civil como espacio de educación no formal y sujeto de acción social que, con su práctica, intenta influir en la dinámica social, educativa, política, económica y cultural del contexto donde se desarrolla (González, 2002).

En América Latina, el origen de la educación no formal está ligado a la educación popular impulsada desde los años setenta y con auge entre las décadas de los ochenta y noventa con las aportaciones especialmente de Paulo Freire (Martín, 2013). Esta relación une los espacios de educación no formal con una propuesta de pedagogía crítica para la lucha y transformación social. En el caso de Chiapas, los espacios de educación no formal experimentaron un parteaguas a partir de 1994 con el movimiento zapatista. Antes del levantamiento del Ejército Zapatista de Liberación Nacional, las asociaciones civiles trabajaban para superar la situación pauperizada de las poblaciones indígenas y aminorar la exclusión social de algunos grupos a partir de la mejora en cuatro ámbitos: la educación, la salud, la defensa del territorio y las condiciones de comercialización y producción, que se establecieron como líneas de trabajo en el I Congreso Indigenista de 1974 llevado a cabo en San Cristóbal de Las Casas (González, 2002).

Durante el conflicto zapatista, numerosas asociaciones civiles se aglutinaron con el objetivo primordial de detener la guerra, denunciar la violación de derechos humanos de la población civil y promover diálogo entre las partes en conflicto (García, 1998). En especial, muchos de estos espacios surgieron con el afán de dar apoyo a las demandas del movimiento zapatista y colaboraron económicamente en algunos de los proyectos de educación formal autónoma-crítica que se consolidaron en ciertas comunidades como una alternativa a las instituciones educativas oficiales del Estado (Gutiérrez, 2006).

Después del movimiento zapatista, las asociaciones civiles dedicadas a la educación no formal quedaron en un lugar más visible y posicionadas muchas de ellas desde la crítica al gobierno y la lucha por el cumplimiento de derechos (González, 2002). En la actualidad, varios de estos espacios desarrollan actividades relacionadas con un amplio abanico de temas, como la promoción de la salud, la defensa del territorio, la cooperación nacional e internacional, la conservación del medioambiente y del patrimonio cultural, la reflexión de género y actividades feministas, el fomento y desarrollo rural y comunitario, además de la defensa de los derechos humanos. Un ejemplo de esto último es la agrupación denominada Red por los Derechos de la Infancia y la Adolescencia en Chiapas (REDIAS), que se constituyó en 2012 con la participación de nueve asociaciones civiles que pretenden impulsar mecanismos, programas y leyes en el estado chiapaneco que contribuyan al cumplimiento de los derechos de menores especialmente indígenas (REDIAS, 2012).

En San Cristóbal de Las Casas se encuentran desde hace dos décadas una gran variedad de asociaciones civiles que han proliferado durante y después del conflicto zapatista con distintos intereses. Sin embargo, ante esa propagación, acrecentó también un debate sobre algunos de estos espacios y la influencia asistencialista y corporativista neoliberal contenida en ellos (Bertely-Busquets, 2016), y es que, en la primera década del siglo XXI, se piensa que muchas asociaciones civiles se han vuelto un elemento necesario en la agenda pública neoliberal al tratar los problemas sociales creados en parte por los recortes en políticas públicas (González, 2002). Por ello, sigue vigente el cuestionamiento crítico sobre el ejercicio 
que algunas de estas llevan a cabo en la región; es decir, surgen dudas sobre sus discursos y acciones que en algunos momentos podrían parecer contradictorios en la práctica:

Este auge de las ONG's responde a las políticas neoliberales de adelgazamiento del Estado, mediante las que se pretende relegar sus obligaciones sociales en manos de la iniciativa privada, en las fuerzas del mercado, por lo que podría pensarse que el zapatismo simplemente le ha facilitado las cosas al gobierno mexicano [...] Éstas, buscan presionar para que el Estado asuma sus obligaciones en la materia, pero respondiendo a los requerimientos de sus destinatarios en un marco de relaciones complejo y contradictorio (dialéctico) (Gutiérrez, 2006, p. 104).

Ante esto último, se encuentra un paralelismo entre la consolidación de posturas críticas que discuten la función de estos espacios y un debate intercultural crítico que también se abre paso en América Latina de manera social, teórica, epistémica y política para reivindicar otras formas pedagógicas y relacionales (Walsh, 2002; Gunther y Cortés, 2011; Bertely-Busquets, 2016). A continuación, retomamos este debate para explicar cómo la interculturalidad crítica se convierte en una herramienta para esta investigación con la que se reflexiona la experiencia en Amalgama, AC (a partir de aquí Amalgama), que representa uno de estos espacios de educación no formal que trabaja con niños, niñas, adolescentes y sus familias de origen indígena en San Cristóbal de Las Casas, Chiapas, México.

\section{HACIA EL PROBLEMA DE INVESTIGACIÓN, PREGUNTAS Y OBJETIVOS: LA INTERCULTURA- LIDAD CRÍTICA PARA PENSAR LA EXPERIENCIA EN AMALGAMA}

A partir de la mitad del siglo XX, la interculturalidad se retomó desde los movimientos indígenas en América Latina para superar los límites del multiculturalismo debido a que este es considerado como una ideología que reconoce y teoriza sobre el pluralismo cultural, pero es indiferente con las diferencias y las formas injustas de relación entre las culturas (Bauman, 2003).

No obstante, el concepto de la interculturalidad, a lo largo de los años, también queda en parte atrapado por un discurso funcional al sistema (Turbino, 2004). En las últimas décadas, la interculturalidad se ha empezado a reconocer constitucionalmente por países de América Latina, como en México y Ecuador, que aterrizan su reconocimiento en distintas políticas educativas y sociales (Walsh, 2010). Sin embargo, esta situación impulsó posturas críticas que denunciaron la ineficiencia del reconocimiento de la interculturalidad si no había cambios relacionales más profundos (Turbino, 2004; Walsh, 2009c; Estermann, 2014).

Podemos decir que la interculturalidad en su perspectiva crítica surge para defender que la lucha contra la desigualdad o la exclusión no omita el problema de la estructura colonialista-capitalista-patriarcal que tiene su efecto en formas estructurales, pero también institucionales y relacionales (Turbino, 2004; Walsh, 2009c). Por ello, traslada el debate de lo intercultural más allá del ámbito de la educación o del marco jurídico constitucional, y lo extiende a cuestionamientos ontológicos, epistémicos y existenciales: "La interculturalidad crítica debe ser entendida como una herramienta pedagógica, que pone en cuestionamiento continuo la racialización, subalternización e inferiorización y sus patrones de poder, visibiliza maneras distintas de ser, vivir y saber" (Walsh, 2010, p. 22). 
Abundan estudios sobre los usos de la interculturalidad en la educación formal, pero no en los espacios de educación no formal; por ejemplo, son reconocidos los estudios en México, y en particular en Chiapas, acerca de las propuestas de las universidades interculturales (Dietz, Selene y Mateos, 2002; Schmelkes, 2003; Sartorello, 2009). Estos estudios configuran un análisis del contexto en que fueron creadas las universidades y una evaluación crítica como un modo de reconocer qué tan "interculturalizadas" están; esto, en especial, después de implementar el programa educativo intercultural o la educación intercultural bilingüe. Sin embargo, existen pocos estudios que trasladan el marco referencial de la interculturalidad crítica a los espacios de educación no formal que plantean superar la exclusión social de ciertos grupos.

Este sería uno de los aportes de nuestra investigación, ya que se efectúa una integración de la interculturalidad crítica en los espacios de educación no formal, en sus relaciones y su práctica socioeducativa. Esta última, aunque aparece como término en singular, está conformada por todos los discursos y las acciones (visión, misión, objetivos, cursos, talleres, conferencias, foros, materiales educativos, etcétera) que una asociación civil de educación no formal realiza para cumplir con lo que se propone en relación con el grupo al que los dirige. En el programa de Amalgama, la práctica socioeducativa no se centra exclusivamente en el apoyo educativo; también ofrece capacitaciones en oficios, contenidos en desarrollo humano, acompañamiento a madres y padres, creatividad, arte y deporte a niños, niñas, adolescentes y sus familias de origen indígena.

Para pensar el espacio, las relaciones y la práctica socioeducativa de Amalgama, profundizamos en la noción de estructura colonial-capitalista-patriarcal (De Sousa, 2003; Maldonado, 2003; Walsh, 2010; Lugones, 2011; Grosfoguel, 2013), que estaría configurada por elementos como la colonialidad, las relaciones de poder, la discriminación, la dominación y el patriarcado (Rubin, 1986; Foucault, 1988; Gasché, 2008; Walsh, 2009a; Comboni y Juárez, 2013; Estermann, 2014). Retomar estos elementos se considera esencial porque, si bien muchos de estos espacios se fundaron partir del movimiento zapatista para la transformación social del problema de la exclusión, algunos pueden estar causando efectos contradictorios ante ese problema en el espacio, las relaciones y la práctica socioeducativa que llevan a cabo.

En esta investigación replanteamos que el problema de la exclusión no se resuelve promoviendo estrategias de adaptación o inclusión de unos grupos excluidos, sino que exige también la visibilización de esta estructura colonial-capitalista-patriarcal en todos los ámbitos y la posibilidad de desmontar los hitos que la conforman. En concreto, a efectos de este artículo, profundizamos en la colonialidad en sus tres ejes: del ser, del poder y del saber desde la experiencia en Amalgama.

El objetivo general es repensar desde la interculturalidad crítica a Amalgama, que como sujeto de acción social pretende superar la exclusión de niños, niñas, adolescentes y sus familias de origen indígena que viven en el municipio de San Cristóbal de Las Casas. Para ello, dado que nadie aprende o enseña desde un no lugar (Arroyo et al., 2016), reflexionamos la situacionalidad de ese espacio y formulamos las siguientes preguntas de investigación: ¿desde dónde se sitúa Amalgama como sujeto de acción social para superar la exclusión de niños, niñas, adolescentes y familias de origen indígena?, ¿qué práctica socioeducativa realiza 
esta asociación para superar la exclusión en San Cristóbal de Las Casas, Chiapas, México?, y ¿̇cuáles son las experiencias de las personas-sujeto que participan en los encuentros educativos desde Amalgama?

\section{Metodología}

El diseño de la investigación se basa en el paradigma sociocrítico con el que nos propusimos interactuar con el fenómeno social para incidir en la transformación por medio de la reflexión crítica y la participación de las personas involucradas (Del Rincón, Arnal, Latorre y Sans, 1995). El paradigma sociocrítico difiere del positivista o el interpretativo por su incidencia en la realidad desde una postura crítica y dialógica-transformadora (Colmenares, 2012); es decir, no se limita a indagar en la realidad para conocerla o interpretarla, sino que se enfrenta a ella de manera crítica y repiensa sus estructuras y relaciones sociales para transformarlas.

En esta investigación abordamos el fenómeno de la exclusión desde la experiencia en Amalgama y la posibilidad de transformar espacios, relaciones y prácticas socioeducativas que afectan la permanencia del problema o el fenómeno: es un estudio que le preocupa la problemática de la exclusión en los espacios de educación no formal y abre la posibilidad de una discusión transformadora.

La metodología fue de corte cualitativo, lo que facilitó adentrarnos en la profundidad y subjetividad de la realidad, las personas y las relaciones; supera la interpretación del problema social para profundizar en un debate crítico (Taylor y Bogdan, 1987). El enfoque es teórico-metodológico, fundamentado en la herramienta de la interculturalidad crítica. La propuesta de integración metodológica desde la interculturalidad crítica busca develar elementos de la estructura colonial-capitalista-patriarcal e indagar en la posibilidad de lo decolonial para superarlos desde la experiencia en un espacio de educación no formal.

También es interés de este artículo la visibilización y discusión de la colonialidad en la práctica socioeducativa de Amalgama, así como la posibilidad de transformación con una aproximación a lo decolonial desde algunas de las experiencias de las personas-sujeto educadoras. El término "persona-sujeto" que aparece a lo largo del texto hace referencia, por un lado, al concepto de "sujeto social" utilizado habitualmente en las reflexiones que parten de la pedagogía del sujeto (Larrosa, 2003; Rivas, 2005; Bárcena, Larrosa y Mélich, 2006; Zemelman, 2010; Berlanga, 2014a) y, por otro, destaca en lo "personal" y singular de un "sujeto", que también afecta el encuentro pedagógico.

\section{Investigación en Amalgama}

Nos centramos en el discurso y la acción oficial de Amalgama, que conocimos a partir de la experiencia compartida por la persona fundadora, así como de la revisión de documentos que nos proporcionó la asociación. También, incluimos testimonios de las personas-sujeto que coinciden durante el encuentro pedagógico, es decir, algunas de las educadoras que dirigen la práctica socioeducativa y algunos niños, niñas, adolescentes, madres y padres que asisten al espacio.

Amalgama es el nombre ficticio que representa el verdadero espacio donde se realizó la investigación. A lo largo del contenido de este documento, mantendremos el anonimato de las personas participantes y no revelaremos datos que puedan identificar el verdadero nombre. 
La asociación está ubicada en el municipio de San Cristóbal de Las Casas, Chiapas, México, y trabaja con niños, niñas, adolescentes y sus familias de origen indígena con enfoque en los derechos humanos. Las edades de quienes llegan a la asociación van de los seis a los catorce años. Las familias, en su mayoría, son originarias de comunidades aledañas a San Cristóbal de Las Casas y muchas de ellas se asentaron en la periferia del municipio debido a algunas expulsiones religiosas en diferentes comunidades cercanas y migraciones antes y después del movimiento zapatista (Uribe y Martínez, 2012).

El contenido de su programa se centra en la defensa de los derechos humanos y se organiza en tres áreas: área académica, con base en el apoyo de las tareas escolares, lectura en biblioteca, reforzamiento del pensamiento lógico mediante el ejercicio lúdico y creativo; el área de desarrollo humano, con actividades para el autocuidado, el espacio de escucha, las tutorías y los talleres con padres y madres; y el área artística, creativa y de deporte, con actividades para desarrollar labores plásticas, corporales y visuales para la expresión de emociones y experiencias.

\section{Etapas de investigación}

Las etapas de investigación se consideran como "situaciones" que aluden la importancia de la situacionalidad en las investigaciones sociales y la interculturalidad crítica (Harding, 1996; Mèlich, 2008; Walsh, 2017). Estas fueron distintas y dependen de cómo la persona que investiga decide posicionarse y reflexionar en el espacio y con las personas-sujeto.

Tabla. Etapas de investigación

\begin{tabular}{|c|c|c|c|c|}
\hline $\begin{array}{c}\text { Situación o } \\
\text { etapa }\end{array}$ & ¿Desde dónde? & Técnicas & ¿Con quién? & ¿Para qué? \\
\hline $\begin{array}{l}\text { Autorre- } \\
\text { flexión } \\
\text { crítica }\end{array}$ & $\begin{array}{l}\text { Persona-sujeto } \\
\text { reflexiva-crítica } \\
\text { de la práctica }\end{array}$ & $\begin{array}{c}\text { Observación } \\
\text { participante } \\
\text { Diario de } \\
\text { campo } \\
\text { Revisión de } \\
\text { documentos }\end{array}$ & $\begin{array}{l}\text { Con las } \\
\text { personas- } \\
\text { sujeto en el } \\
\text { encuentro } \\
\text { pedagógico }\end{array}$ & $\begin{array}{c}\text { Elementos de la } \\
\text { estructura capitalista- } \\
\text { patriarcal } \\
\text { Reflexiones sobre la } \\
\text { práctica socioeducativa } \\
\text { en primera persona }\end{array}$ \\
\hline $\begin{array}{l}\text { Encuentro } \\
\text { individual }\end{array}$ & $\begin{array}{l}\text { Desde la escu- } \\
\text { cha a las otras } \\
\text { personas-sujeto }\end{array}$ & $\begin{array}{l}\text { Entrevistas } \\
\text { Diario de } \\
\text { campo }\end{array}$ & $\begin{array}{l}\text { Equipo de } \\
\text { trabajo } \\
\text { Madres de la } \\
\text { asociación }\end{array}$ & $\begin{array}{c}\text { Elementos de la } \\
\text { estructura capitalista- } \\
\text { patriarcal } \\
\text { Reflexiones sobre la } \\
\text { práctica socioeducativa } \\
\text { de otras personas }\end{array}$ \\
\hline $\begin{array}{l}\text { Reflexión } \\
\text { grupal }\end{array}$ & $\begin{array}{l}\text { Desde la escu- } \\
\text { cha de las otras } \\
\text { personas-sujeto }\end{array}$ & Grupos focales & $\begin{array}{c}\text { Niños, niñas } \\
y \\
\text { adolescentes } \\
\text { Equipo de } \\
\text { trabajo }\end{array}$ & $\begin{array}{l}\text { Reflexiones conjuntas } \\
\text { sobre la práctica } \\
\text { socioeducativa }\end{array}$ \\
\hline
\end{tabular}


En una primera etapa, la persona que investiga se sitúa en campo como observadora participante en las prácticas socioeducativas y como reflexiva-crítica del sentir en esa situación a través de la redacción de un diario de campo. En esta etapa se produjo una triangulación de la información de lo que observamos en el espacio, las relaciones y la práctica socioeducativa con los documentos revisados y las experiencias compartidas de las personas que participan en la asociación. Ahí, obtuvimos elementos de la estructura colonial-capitalista-patriarcal y las primeras reflexiones sobre la asociación escritas en primera persona, es decir, autorreflexiones de la persona que investiga.

En una segunda etapa, llevamos a cabo los encuentros individuales con otras personas-sujetos en calidad de entrevistas semiestructuradas. La guía de entrevistas se centró en otorgarle importancia a la experiencia de las personas-sujeto en la asociación; por ello, partimos de preguntas abiertas cómo ¿cuál es un día normal para ti en la asociación?, ¿cuáles han sido tus aprendizajes?, ¿cuáles son las dificultades que enfrentas? y también, ¿cómo ha cambiado tu práctica desde tu primer día hasta ahora? y ¿qué es lo que mejorarías de tu práctica?; esto, en el caso de las personas educadoras.

En estos encuentros, la persona que investiga se posicionó desde la escucha. Durante esta situación aparecieron más elementos de la estructura colonial-capitalista-patriarcal y, sobre todo, surgieron reflexiones sobre la práctica socioeducativa expresadas y compartidas por otras personas-sujetos educadoras, niños, niñas y adolescentes y algunas madres. Estas reflexiones revelaron emociones y sentimientos, así como dificultades, facilidades, esperanzas, retos y aprendizajes de las propias personas-sujeto, especialmente educadoras, que compartieron sus experiencias en el espacio y en las relaciones de Amalgama. Esta etapa reveló la complejidad del espacio, las relaciones y la práctica.

La tercera situación tuvo lugar en los espacios de reflexión conjunta de los grupos focales en los que se profundizó en las complejidades del contexto, en el sentir de cada persona-sujeto educadora, en el espacio de educación no formal como encuentro pedagógico y en las dificultades dentro de la asociación para realizar su práctica.

Todas las etapas se desarrollaron de una manera continua y colaborativa durante la investigación.

Categorías de análisis: una reflexión de la colonialidad y sus efectos en el encuentro pedagógico

El proceso de análisis inició con la selección de las observaciones y notas de campo de discursos representativos de las personas participantes y de los documentos oficiales de la asociación, lo que constituyó un ejercicio de codificación. Esta primera selección ofreció la posibilidad de ir construyendo categorías de análisis que vincularon la información desde la experiencia en campo con la teoría (Coffey y Atkinson, 2003). La codificación facilitó el reconocimiento de algunos elementos de la estructura colonial-capitalista-patriarcal en Amalgama, que en este artículo se centrarán en la colonialidad en sus tres ejes: ser, saber y poder.

La forma en que se operacionalizaron estos ejes de la colonialidad tuvo que ver, por ejemplo, en el caso de la colonialidad del ser con cuestiones como "la inferiorización de otras personas y sus vidas por su adscripción cultural y usos y costumbres" o "prácticas socioeducativas dirigidas a transformar los comportamientos entre o al educando o entre el equipo de trabajo por no ser apropiadas". 
En cuanto a la colonialidad de poder, esta se operacionalizó atendiendo, por ejemplo, "el imperativo de normas conductuales para establecer las conductas estándar" o "los criterios de selección para el personal, familia y voluntariado de la organización". La colonialidad del saber se operacionalizó de acuerdo con cuestiones como "la percepción jerárquica del saber occidental como superior a otros saberes" o "las prácticas socioeducativas desarrolladas a partir de criterios de la educación oficial", entre otras.

Las categorías cobraron sentido in situ debido a la experiencia directa en el espacio y con las personas que participaban en la práctica socioeducativa. De esta manera, en el trabajo de campo, realizando las observaciones participantes, las entrevistas y los grupos focales, emergieron categorías de análisis nuevas, es decir, categorías in vivo, además de que algunas de las categorías a priori empezaron un proceso de transformación. Del mismo modo, durante el proceso de codificación, después de volver a revisar la teoría y efectuar un ejercicio de asociación entre ellas, aparecieron nuevas categorías y otras sufrieron transformaciones.

Para el análisis de la información, utilizamos como herramienta de apoyo, en primer lugar, el programa QSR en la versión N6, que facilitó la organización de la información en códigos y categorías. En segundo lugar, elaboramos un mapa explicativo que permitió asociar las categorías con el espacio, las relaciones y la práctica socioeducativa. Este ejercicio de asociación nos permitió conocer la relación de las categorías de la interculturalidad crítica con el espacio de la educación no formal y descubrir la complejidad de las relaciones sociales que develaban cómo se manifiesta la estructura colonial-capitalista-patriarcal en el espacio, las relaciones y la práctica socioeducativa en Amalgama. Así, emergieron las categorías "práctica de intervención", "tensiones", "colonialidad del ser", "colonialidad del saber", "colonialidad del poder", "(des)situación" y "práctica de aproximación a lo decolonial”, que abordaremos en este artículo.

\section{Ética de la investigación}

Para nuestra investigación, requerimos un permiso de Amalgama para participar en su espacio y en sus actividades. También fue imprescindible que la asociación conociera los objetivos del estudio. Se suscribió un formato de consentimiento previo informado antes de la entrevista a cada persona, el cual constaba que la participación de los sujetos era libre, voluntaria y que no ocasionaría daño de ningún tipo. De la misma manera, antepusimos la confidencialidad y la protección de las personas al no exponer sus nombres ni datos de identificación.

\section{RESULTADOS Y DISCUSIÓN: REFLEXIÓN DE LA PRÁCTICA DE INTERVENCIÓN Y LA COLONIALIDAD EN AMALGAMA}

Después de analizar la información proveniente del trabajo de campo y contrastarla con la teoría, exponemos los resultados, los cuales reflejan que la utilización de la herramienta de la interculturalidad crítica sirve para develar cómo la colonialidad se encuentra de manera sutil en el espacio, en las relaciones y en la práctica de Amalgama. Esto invita a reflexionar cómo la estructura colonial-capitalista-patriarcal sucede en una "práctica de intervención" hacia niños, niñas, adolescentes y sus familias de origen indígena que, aunque quiere solventar el problema de la exclusión, puede llegar en ciertos momentos a reproducirla. Sin embargo, demuestra que es posible encontrar lo decolonial en la "(des)situación" 
de las personas-sujeto educadoras, lo que nos acerca a pensar también en una aproximación a lo decolonial en Amalgama.

\section{Re-pensando la colonialidad del ser}

Amalgama es una asociación civil que se consolida por el ímpetu de las personas fundadoras para responder a la urgencia social indígena reconocida por el movimiento zapatista:

Yo estando en [país de Europa] digo: "Pues yo quiero regresar a México". Te estoy hablando del 94, 95, cuando se levanta el movimiento zapatista, es cuando yo me voy a [país de Europa]. Entonces me empiezo a preguntar: “¿Qué estoy haciendo aquí?" Conozco a mi pareja también con sus intereses que él tenía personales de su proyecto de vida y coincidimos en hacer esto juntos. Es decir, de venir a México y hacer una experiencia en una organización que trabajara con infancia y con mujeres indígenas (persona fundadora).

Desde los inicios de esta asociación civil se concibe la necesidad de impulsar una práctica socioeducativa hacia la infancia con menos recursos económicos de San Cristóbal de Las Casas, en un periodo que se nombró por el equipo de trabajo durante el grupo focal como "visión de una infancia libre de pobreza, explotación laboral, violencia y discriminación, capaz de construir sueños":

$Y$ en aquel entonces era muy común que los niños estuvieran con su libretita y decían: "Fírmame y ayúdame para mis útiles" [...] Y cuando nosotros empezamos a preguntar: “¿Vas a la escuela? ¿Por qué no vas a la escuela?". Para mí fue muy decepcionante ver que muchos niños decían que no iban a la escuela porque no querían (persona fundadora).

En 2005 se constituyó oficialmente el espacio de Amalgama como asociación civil con una misión, visión y objetivo principal:

A. Visión: infancia libre de pobreza, explotación laboral, violencia y discriminación capaz de construir sueños (grupo focal).

B. Misión: a través del desarrollo educativo, las niñas y niños aprenden herramientas para transformar su entorno, aprenden a ser conscientes de sí mismos y se preparan para poder salir de la pobreza de forma autosuficiente. Para poder alcanzar esta misión las niñas y niños reciben una educación con enfoque en los derechos humanos de la infancia (tríptico informativo).

C. Objetivo principal: empoderar a niños, niñas y jóvenes de las colonias marginadas de San Cristóbal de Las Casas (Informe anual Amalgama, 2015-2016).

Tanto la visión, la misión como el objetivo principal de la asociación se dirigen a trabajar con niños, niñas y adolescentes que, según el discurso, se ven afectados por la exclusión, marginación o pobreza y sufren determinadas carencias:

Los niñas, niñas y adolescentes que apoya Amalgama provienen en gran parte de familias numerosas. La mayoría de sus padres carecen de educación y de un trabajo digno. [...] Muchos de estos niños y niñas se ven con la necesidad de apoyar 
a su familia económicamente y trabajan como boleros, limpiadores de parabrisas, dulceros, vendedores de artesanías, sin poder ir a la escuela. Bajo estas condiciones no pueden terminar una formación escolar y no tienen las herramientas necesarias para salir, por sí solos, de este círculo de marginación y pobreza [...] Por otra parte, no existen alternativas en sus colonias marginadas para que realicen actividades provechosas en su tiempo libre (tríptico informativo de Amalgama).

En concreto, se habla de carencias en el discurso oficial que suceden especialmente en la periferia norte de la ciudad, la cual presenta déficits como el de inclusión con el resto de la ciudad o el de planeación territorial, que ocasionan bajo nivel escolar o delincuencia. De esta manera, se nombra a los niños, niñas y adolescentes que llegan al espacio como una población-víctima: víctima de la estructuraEstado, que no les garantiza sus derechos; víctima del sistema educativo por su mala calidad; y principalmente, víctima de la periferia donde viven sus familias en un contexto pobre y violento:

El $90 \%$ de la población con la que trabajamos son niñas, niños y adolescentes de las periferias de la ciudad donde se acentúa la marginación y la pobreza. Las características de las colonias en la periferia son su origen de asentamiento irregular y el constante crecimiento hacen que tengan un difícil acceso y una deficiente planeación territorial. Los principales problemas señalados en estos territorios son el bajo nivel de escolaridad, el empleo poco remunerado de sus habitantes y el alto índice delictivo (Informe anual Amalgama, 2015-2016).

Nos adentramos, así, en la discusión de un elemento importante en la estructura colonial-capitalista-patriarcal, que es la colonialidad del ser (Maldonado, 2003; Mignolo, 2005; Walsh, 2005). Retomamos la colonialidad del ser en su relación con la interiorización de la subalternalización y deshumanización de los otros sujetos (Mignolo, 2005; Walsh, 2009c). Este tipo de colonialidad guarda relación íntima con el lenguaje cuando se alude a unas formas de ser y estar que son más válidas que otras (Maldonado, 2003); es decir, la colonialidad del ser se identifica en el discurso de la asociación cuando emplea el uso directo de un tipo de lenguaje: "La mayoría de sus padres carecen de educación y de un trabajo digno" o "No existen alternativas en sus colonias marginadas".

Por otro lado, la colonialidad del ser se refuerza también en acciones concretas, por ejemplo, al imponerse normas y acuerdos en el espacio que determinan formas concretas de estar frente a otras que no son válidas: "No comer hablando, cuido la comida que me den, guardo la fila, me lavo los dientes después de comer, mastico con la boca cerrada, si hablo no mastico, si mastico no hablo, me mantengo sentad@ mientras como mis alimentos o me lavo las manos" (cartel de normas de la asociación).

El cartel anterior simboliza cómo la asociación considera que hay ciertos comportamientos adecuados sobre otros que no lo son; hay un compromiso específico en torno a la higiene y las familias deben cumplir con cuidar el aseo e higiene personal de los niños y las niñas: "Me bañaré en casa, vendré con las uñas cortadas, asistiré con mi ropa y calzado limpio, si tengo piojos los atenderé para eliminarlos y evitar posibles contagios" (Carta de compromisos 2016).

Lo anterior muestra la relación de este tipo de espacios de educación no formal con la tendencia higienista de tradición europea, la cual, en particular desde el 
siglo XIX, queda asociada a espacios de educación que trabajan con población en contextos de marginación y pobreza (Viñao, 2010).

En el caso concreto de este espacio de educación no formal, la práctica socioeducativa se vuelve cercana a una de intervención, que consiste en intervenir en los niños, niñas, adolescentes y familias que llegan a la asociación para que transformen su propia vida. La idea de intervención se retoma de Berlanga (2014a), quien la define como "la relación educativa que se piensa y se construye desde afuera" y desde ese lugar, desde fuera, se identifican los problemas o las carencias en las otras personas a partir de los cuales serán intervenidas.

Esto último es el acto que deshumaniza el encuentro, porque las personas quedan tematizadas como población-víctima víctima (Giner, Saldívar, Duarte y Keck, 2017); es decir, la práctica de intervención se basa en la noción de la periferia como negativa que caracteriza a estos niños, niñas y adolescentes; sin embargo, cuando ellos hablan de su propia identidad y de las relaciones sociales en San Cristóbal de Las Casas, no surge la noción de carente:

\begin{abstract}
Hay muchos gringos, estos son güeros, pelo rubio y turistas [...] también están los indígenas, como dicen los criollos, mestizos o coletos, a los que no son criados aquí, muchos coletos son racistas [...]. El turismo nacional e internacional compra a los indígenas, les vende más caro a ellos porque tienen más dinero [...], pero los indígenas tenemos más cultura que los turistas, tenemos terreno, flora y fauna y zonas rurales (testimonio de niños, niñas y adolescentes).
\end{abstract}

Estas reflexiones que surgieron en el grupo focal con los niños, niñas y adolescentes revelan que no son sujetos pasivos ante estas cuestiones sociales, sino que, en su mayoría, son niños, niñas y adolescentes reflexivos y críticos. La concepción de población-víctima por parte de la asociación queda en entredicho cuando los niños, niñas y adolescentes reconocen sus propios recursos como positivos. Compartimos el testimonio de una madre en referencia a sus hijos que asisten a la asociación:

Mis hijos nacieron aquí en San Cristóbal de Las Casas, pero veo que mis hijos se sienten orgullos de que son de allá también [una comunidad de los Altos de Chiapas]. Para ellos, ellos son de allá, porque yo soy de allá y se sienten así. Eso, me sorprende porque pienso que creo es a mí a la que están dando lecciones ellos mismos.

La práctica socioeducativa como práctica de intervención plantea desde un inicio una relación que se establece en algunos momentos como de autoridad de quien la imparte hacia quien la recibe: "te voy a formar", "te voy a educar", "te voy a integrar", o "te voy a incluir", lo que condiciona las relaciones sociales en el espacio (Berlanga, 2014a). Ello genera que Amalgama, como sujeto de acción social, reproduzca una práctica de intervención hacia el otro que devela colonialidad en el espacio, en las relaciones y en la práctica, lo cual ocasiona la exclusión de formas de estar y ser frente a otras que se imponen como más válidas.

\title{
RE-PENSANDO LA COLONIALIDAD DEL SABER Y EL PODER
}

En este apartado discutimos la colonialidad del saber y el poder en el espacio, las relaciones y la práctica socioeducativa al retomar información de las evaluaciones 
que se basan en indicadores definidos en el marco lógico (ML). A lo largo de los años, la asociación civil estableció ciertos indicadores de éxito descritos en el ML que son determinados por el país europeo financiador. El ML es un método de evaluación de proyectos generalmente utilizado por las agencias de cooperación al desarrollo (como el Banco Mundial o el Banco Interamericano) con el objetivo de lograr resultados concretos en un periodo y en una zona geográfica para resolver los problemas de un grupo (Camacho, Cámara, Cascante y Sainz, 2001). Amalgama, de acuerdo con el ML, impulsó con más fuerza el desarrollo educativo y el enfoque hacia los derechos humanos y redujo sus alcances en indicadores y porcentajes:

- Las y los participantes concluyen la primaria y se insertan en la secundaria. $50 \%$ de los participantes tiene el conocimiento matemático según nivel escolar. $75 \%$ el promedio de las niñas y niños mejora o es mayor de 8.0.

-Participantes conocen sus derechos y responsabilidades, los cuales ejercen con autosuficiencia.

70\% Conoce sus 5 derechos básicos.

$50 \%$ Reconoce cuáles son sus responsabilidades relacionadas con la familia y escuela (en la familia: tender la cama, cocinar, secar platos, guardar la ropa limpia; en la escuela: realizar tareas, poner atención al maestro, acomodar lápices, colores y sillas; en la AC: cepillarse los dientes, realizar las tareas, escuchar al tutor, lavar los trastes que usamos, cuidar a nuestros compañeros, acomodar lo que utilizamos) (Informe anual Amalgama, 2015-2016).

La colonialidad del saber se mantiene por el posicionamiento de determinados saberes como superiores desde el orden racional-occidental conocimiento y pensamiento (Lander, 1993). En este sentido, esta colonialidad organiza marcos epistemológicos, academicistas y disciplinares y desacredita otras formas de conocer o de pensamientos propios de muchos pueblos (Walsh, 2009a, 2009c). En Amalgama hay una ausencia de indicadores que aluden a elementos de la cosmovisión indígena; por ejemplo, se prioriza aprender matemáticas y castellano, pero no se nombra en el informe la posibilidad de mantener conocimientos sobre el tsotsil, que es la lengua materna de la mayoría de estos niños, niñas y adolescentes que asisten a la asociación: "Durante la sesión, la persona coordinadora ha explicado a otras educadoras que hay que mejorar aún más el índice en castellano" (diario de campo, semana 3).

Sucede lo mismo con la práctica de intervención cuando se enfoca principalmente a los derechos humanos. En el grupo focal con el equipo de trabajo se destacó como un hito determinante que la asociación civil se uniera a REDIAS, al intensificar su defensa por los derechos humanos y de la infancia en el municipio. En la discusión, la definición brindada acerca de lo que significa "derechos" fue la siguiente: "Se relaciona con las normas y leyes para la convivencia, para el desarrollo de los niños, niñas y adolescentes con los que trabaja Amalgama para su libertad y su vida digna garantizando su ejercicio como sujeto de derechos" (grupo focal con el equipo de trabajo).

Ante esto, la persona fundadora explicó lo importante que es para la asociación ser una institución "vigilante de derechos": "En ese sentido, si regresamos al enfoque de derechos de infancia, no olvidaría que está el Estado, las organizaciones y el adulto. [...] Nosotros desde Amalgama asumimos un rol de observadores vigilantes de los derechos de los niños y las niñas". 
Lo anterior permite reflexionar cómo un espacio de educación no formal, como sujeto de acción social, se posiciona desde el enfoque de derechos humanos. Si se retoma la tradición de los derechos humanos, esta se inspira en gran medida en la Declaración de los Derechos del Hombre y del Ciudadano de la Revolución Francesa en 1789, y aunque se plantearon en su origen como exclusivos del hombre -y del hombre blanco burgués-, hoy se promulgan como símbolo sine quan non humano a partir de la Declaración Universal de los Derechos Humanos (1948).

El debate del universalismo de los derechos humanos aparece, sin embargo, para cuestionar la forma en que se extiende el discurso de los derechos humanos (Pérez, 1998; Peces-Barba, 2002), que tiene relación con el pensamiento abismal que propone De Sousa (2009), con base en el cual existe una línea que se convierte en una frontera de pensamiento que rara vez se cuestiona, dado que significa transgredir lo que legaliza una forma de ser, conocer y estar en el mundo. Los derechos humanos y ciertos conocimientos, en cuanto a su concepción como universales, estarían planteados desde ese lado de lo existente y reconocidos como válidos de manera universal: "La universalidad de los derechos hace referencia a derechos universales, en el sentido de racionales y válidos para todos los hombres, pero situados en un contexto histórico o geográfico" (Peces-Barba, 2002, p. 615).

De esta manera, se relaciona con la colonialidad del saber porque repercute ontológica y epistemológicamente en la cuestión del conocimiento en el espacio, las relaciones y la práctica socioeducativa de la asociación respecto a cierta negación de los sujetos en la construcción de ese conocimiento (Zemelman, 2010). Amalgama alude a la libertad de los sujetos y la defensa de los derechos humanos, pero provoca un efecto de falta de historicidad y subjetividad en la medida en que reproduce ciertos conocimientos como universales hacia los niños, niñas y adolescentes.

La aproximación al problema que le afecta a un sujeto, como en este caso es la exclusión, debería ser entendido desde la realidad sociohistórica y la subjetividad social constituyente con la que todos los sujetos viven, narran y sienten esa realidad (Zemelman, 2012). Así, la historicidad de los sujetos y el sentido que estos le puedan dar a los derechos humanos simbolizan la posibilidad de conocer y actuar con y desde la experiencia de la persona en lo que sería un encuentro como práctica de libertad y dialogicidad (Freire, 1967). Sin embargo, esto no siempre sucede en Amalgama; por ello, se evidencian algunas dificultades en la práctica, como las que narra una educadora:

A mí a veces me ha pasado con algunos participantes que es como: ¿Qué importan los derechos? Es como que no miden la importancia de sus derechos: "Ah si el derecho de la salud, ya me lo sé". Entonces a veces está esa dificultad de no medir, de no transmitir la importancia que tienen esos derechos en su vida para que realmente lo tomen en cuenta.

Nos adentramos entonces en una colonialidad del saber, pero también del poder cuando la práctica de intervención se convierte, además, en homogeneizante, porque la noción racional-occidental no concibe una "racionalidad del Otro" (Dussel, 1993). Esto lleva a pensar cómo la práctica socioeducativa de Amalgama tiende a inclinarse hacia la obtención de resultados exitosos para cumplir con el ML impuesto por la financiación internacional. Sin embargo, promueve, en algunos momentos, una práctica de intervención descontextualizada y la exclusión indirecta 
de ciertas familias del espacio. Esto sucedió en especial cuando Amalgama decidió medir el programa en términos de eficacia y eficiencia, cumplir con los indicadores del ML y diseñar una carta de compromiso que exigía la presencia continuada de las familias y sus hijos e hijas para garantizar el éxito de programa:

Asistir de lunes a viernes a las actividades del centro que se ofrecen, realizar mis tareas escolares, mejorar mi promedio y/ mantener un mínimo de 8, recordar a mi madre/padre o tutor que entregue mi boleta cada bimestre, participar activamente y con entusiasmo en las distintas actividades que se ofrecen para mí, cuidar de mi aseo e higiene personal, respetar los acuerdos establecidos por la organización y por cada área, respetarme a mí mismo y a todas y todos mis compañeros, respetar a mis facilitadores y voluntarios/as y ejercer con responsabilidad mis derechos, respetar y difundir los derechos particularmente los de las niñas, niños y adolescentes (Carta de compromiso 2016).

Estos compromisos desencadenan que muchas familias no puedan sumarse a los requerimientos del programa; es decir, las familias "más pobres" son las que más necesidades económicas tienen; por ello, sus hijos e hijas llegan a ser mano de obra necesaria para los ingresos monetarios de la casa. Esto les dificulta la presencia constante en el programa y, por tanto, el cumplimiento de la dinámica del "juego" como explica la fundadora:

Si queremos tener esos resultados pues nos vamos a las familias de bajos recursos, pero no los más pobres, porque si queremos realmente ayudarles, sacarnos a la población de más bajos recursos pues son las personas que tienen muchas dificultades para entrar en ese juego. Van a mandar a sus hijos a trabajar porque no les alcanza, porque es la parte de la pobreza, no tienen de otra, pues van a maltratar a sus hijos de forma violenta porque es la única forma en la que ellos lo han aprendido.

Así, las tensiones entre la asociación y las familias se acaban haciendo más plausibles con el tiempo, hasta el punto de que ha ido cambiado el perfil de la población de ingreso, como lo relata una educadora:

Antes, siento yo que había una población que tenía muchos más problemas de violencia y aquí dentro era como algo muy recurrente. Siento que eso ya no se da tanto. Ya no trabajamos mucho con esa población. Siento que la población ahora no tiene tanto esos problemas de relaciones de violencia intrafamiliar, hay algunos casos, pero no es tanto como antes. Siento que eso la población ha ido cambiando. Se ha ido perfilando, definiendo año tras año el perfil de ingreso.

Ante esto último, se vuelve a retomar la noción de práctica de intervención como una "forma instrumental de desarrollar la acción en términos de relación mediosfines, eficacia, eficiencia" (Berlanga, 2014a, p. 2); es decir, la práctica de intervención conlleva una lógica de rendimiento del encuentro pedagógico en términos de eficacia y eficiencia que tendrá más éxito, por ejemplo, cuantos más niños, niñas y adolescentes "se transformen", a pesar de que pueda ser homogeneizante: "Todavía hay muchos retos, primero hacer que esto que se cree exitoso se vuelva 
un porcentaje, si hablamos de números que haya más chavos y chavas que logren llegar a decir 'yo quiero transformar mi vida' y no caer en las mismas situaciones de vulnerabilidad que vemos que se tienen" (educadora).

Lo anterior invita a la reflexión del eurocentrismo intrínseco en la colonialidad del poder cuando la práctica se dictamina desde la financiación europea que condiciona la práctica socioeducativa de Amalgama, con la pretensión característica de la colonialidad del poder de "modernizar" otras poblaciones (Quijano, 2002). Así, el espacio, las relaciones y la práctica socioeducativa de Amalgama se sustentan en algunos momentos en ideas de lo racional-occidental como el éxito, la eficacia y la eficiencia, que quedan descontextualizadas y pueden provocar exclusión a determinadas familias.

\section{LAS (DES)SITUACIONES COMO APROXIMACIÓN A LO DECOLONIAL}

En este apartado aludiremos a las emociones y los sentimientos que las personas educadoras compartieron respecto a las situaciones que han vivido en la asociación estudiada. Estas reflexiones críticas corresponden a las personas educadoras y revelan la subjetividad con la que interiorizan la tensión de la práctica de intervención y, ante ello, abren una grieta, por la cual se pueden repensar estos espacios y relaciones del encuentro pedagógico en claves decoloniales (Walsh, 2017).

Este tipo de reflexiones críticas surgieron en los grupos focales, entrevistas y encuentros informales en los que las educadoras compartieron sus reflexiones acerca de la práctica que desempeñan y se situaron en un lugar diferente al de la práctica de intervención. En estas experiencias compartidas aparecen subjetividades, reflexiones críticas y la posibilidad de resignificación del espacio, relaciones y prácticas en una aproximación a lo decolonial: "Y como educadores, también eso ¿cómo estamos construidos? Y ¿desde dónde estamos construidos? Por eso creo que una de las dificultades es que estamos construidos desde la perspectiva occidental" (persona educadora).

La manera en que la persona educadora se piensa consistió en que se narra y se reflexiona en una relación diferente con la otra persona -es decir, familias o niños, niñas y adolescentes- y, por tanto, podemos decir que es una situación distinta a la práctica de intervención. Si bien, la práctica de intervención deshumaniza el encuentro pedagógico y reproduce elementos de la colonialidad, desde estas grietas se humaniza el encuentro pedagógico porque las personas educadoras exponen sus recuerdos más emotivos, sienten y comparten sus sentimientos:

He visto mucho, no sé, siento que no es algo cultural sino una cosa humana. A pesar de que puedas ver situaciones que te causen frustraciones es como aprender a no juzgar sino a aprender a aprender el por qué están en esa situación o si el padre o la madre tiene un problema de adicción ¿por qué ha llegado a esto no? 0 ¿por qué las tradiciones es pegar a los hijos y está bien visto? (persona educadora).

Este tipo de reflexiones son para esta investigación (des)situaciones, porque la persona educadora se piensa en una situación diferente a la de la práctica de intervención (Giner et al., 2017). Las (des)situaciones son un acercamiento a concebir formas relacionales y pedagógicas que se aproximan a lo decolonial y a otras maneras de ser, sentir y estar en el mundo (Walsh, 2005; Mélich, 2008; Walsh, 2009c; Comboni y Juárez, 2013). Son emociones que no entraman juicios hacia 
otras personas ni hacia una misma, sino que se basan en la experiencia como aprendizaje y, así, salen a la luz anhelos de tiempos pasados y deseos de mejorar en ciertas prácticas:

Antes hacíamos mucho más arte, hacíamos muchas más cosas de teatro, obras de teatro grande y lo íbamos a presentar en teatros en Zinacantán, o sea, como cosas más creativas. [...] Lo que yo sentí es que hubo un giro y que ahora todo tiene que ser meramente académico. Con resultados, cifras y de porcentajes, que antes no había (persona educadora).

Los testimonios de las personas-sujeto educadoras tienen que ver con lo decolonial porque presentan intenciones de reivindicar y resignificar espacios, relaciones y prácticas en pro de otras formas posibles (Giner et al., 2017). De ese modo, se presenta el acercamiento hacia la(s) pedagogía(s) de la decolonialidad que es denunciativa, feminista, descolonizadora, crítica, integradora de saberes comunitarios, desontologizadora, posicionada desde el conocimiento situado, temporal, histórica, de construcción colectiva, deconstructora, y rescatadora de experiencias (Walsh, 2009a, 2017).

La decolonialidad entiende los encuentros pedagógicos desde posibilidades infinitas-otras, desde subjetivad(es), reflexiones y desde las experiencias (Walsh, 2017). En ese caso, en las experiencias compartidas por las personas educadoras percibimos también un movimiento epistémico y lingüístico "que abre la oportunidad para elaborar una relación con la realidad, con el otro, que no es de intervención sino de trato" (Berlanga, 2014a, p.1). Este movimiento lo encontramos en los testimonios de las educadoras cuando se narran en la problemática:

Entonces, para mí sesenta niños, cuando está llena la casa es como de locos porque es como tengo que escucharlos a todos. Y a mí, algo que no me gustara que pasara aquí en [Amalgama] es que valoráramos más la cantidad que la calidad. Entonces, para mí algo bien importante es que les preguntáramos eso a la organización "¿qué es más importante la calidad o la cantidad?" y preguntárselo a la dirección y al donante "¿qué es lo que tú quieres, una cantidad o una calidad?" 0 si son ambas, entonces, ¿cómo lo haces para mediarlo?

La persona educadora que enuncia de manera crítica las situaciones en las que solo haya dificultad e incertidumbre, concibe una realidad pedagógica mucho más honesta y posible de construir otras formas de relación con el otro:

Existe una estructura, o sea, no estamos fuera como educadores de la estructura económica y social que, finalmente, es occidental en un contexto tan diverso y multicultural. Entonces, de qué manera se da un equilibro para que digamos la defensa de los derechos humanos no sea la imposición de un concepto y en base a ese concepto también el desprecio a hacia otros conceptos u otras visiones del mundo. Y porque esa misma estructura dice que lo "socialmente aceptable" es lo mío y que la práctica del otro es lo que sale de lo normal y de este binomio de "lo bueno, lo malo", "la verdad, la mentira", la división de "conocimiento/saber" (persona educadora). 
Para Berlanga (2014b), esto sería dirigirse hacia "el trato" al enunciar la posibilidad de construir desde el "entre" una relación pedagógica con la otra persona-sujeto y alejarse de la práctica de intervención: "Cuando la relación se piensa y se construye desde el 'entre', desde la intersubjetividad, la relación se piensa y se construye desde la consideración del trato, el trato con el otro y, al hacerlo, la relación se abre al acontecimiento" (Berlanga 2014b, p. 1).

Por lo anterior, esta aproximación a la decolonialidad tiene que ver con "con gritos, grietas y siembras de vida" (Walsh, 2017, p. 12) que son "las afueras y fisuras, donde moran, brotan y crecen los modos-otros, las esperanzas pequeñas" (Walsh, 2017, p. 31). Con base en la experiencia en Amalgama, aparecen categorías de la estructura colonial-capitalista-patriarcal como la colonialidad, pero también existe la posibilidad de encontrar ciertas grietas (Giner et al., 2017). Estas reflexiones críticas que nacen de las subjetividades de las personas pueden abrir la puerta para un replanteamiento decolonial de las prácticas socioeducativas dado que decolonialidad "no es meta o fin en sí mismo sino herramienta política y conceptual que ayuda a vislumbrar la problemática en su complejidad y esclarecer el rumbo" (Walsh, 2009b, p. 234). Encontrar estas grietas en los testimonios de las personas-sujeto educadoras ha sido muy valioso, ya que hace posible confiar en las probabilidades de resignificación en estos espacios, relaciones y prácticas.

\section{CONCLUSIONES}

De acuerdo con los objetivos de esta investigación, hemos presentado una reflexión crítica de la situacionalidad del sujeto de acción social desde Amalgama. Principalmente, hemos reflexionado sobre el espacio, las relaciones y la práctica socioeducativa que se vuelve una práctica de intervención desde su consolidación inicial. Este tipo de práctica ocasiona tensiones con las familias y la reproducción de la colonialidad del ser, poder y saber. Por lo tanto, podría decirse que, al reproducirse estas tensiones y dichos elementos, se vuelve compleja la posibilidad de superar la exclusión social de esta población, porque, más bien, puede reproducirla de modo sutil.

También es importante rescatar que Amalgama concibe a la población que llega al espacio como población-víctima. Esta cuestión parte de justificar la necesidad de consolidar el espacio por las carencias que sufre el grupo con el que se trabaja, pero rara vez se alude a la potencialidad que puede presentar esa población. Podemos aseverar que es una práctica de intervención que nace y se mantiene en la carencia del otro y no tanto en su potencialidad.

La práctica socioeducativa de Amalgama se convierte, además, en una "práctica de depósito", que otorga o deposita la responsabilidad de cambiar la condición de excluido a la otra persona. En ese sentido, el discurso y la acción oficial y las personas-sujeto educadoras que realizan una práctica de intervención y de depósito hacia los niños, niñas y adolescentes se mantienen al margen de dicho problema, porque le "depositan" a la otra persona toda la responsabilidad del cambio al considerar que ellos deben salir de manera autosuficiente de la exclusión en la que viven. En otras palabras, en la práctica de depósito superar el problema de exclusión atañe en forma exclusiva a las personas beneficiarias del programa y pareciera que si no superan su condición de excluidos y pobres "es porque no han querido". Por ello, su problema podría superarse solo cuando aceptan "ser depositados" $y$, con ello, cambian sus formas de estar y ser en el mundo por otras que se plantean como más dignas y válidas. 
Esto último nos lleva a reflexionar sobre la manera en que se entiende la transformación social en dicho espacio, como sujeto de acción social que quiere mejorar el problema de la exclusión de población indígena de San Cristóbal de Las Casas, pero que pudiera caer en contradicciones si les deposita toda la responsabilidad a los propios sujetos participantes, y se olvida de las causas estructurales y relacionales.

Ante esto último, nuestra investigación defiende la posibilidad de comprender la transformación social desde una perspectiva que reivindica el cambio estructural e institucional, pero también relacional. Por ello, la transformación social puede concretarse al cambiar la forma en que nos relacionamos con los demás y con nosotros mismos desde estos espacios. No solo en cómo adaptamos el contenido y la práctica para la "inclusión" del otro. Esto significa que un espacio de educación no formal puede plantear la transformación de la exclusión al concebir nuevos modos de relación con los demás desde el propio espacio, las relaciones y la práctica; es decir, cuestionando cómo nos pensamos con los demás, cómo nombramos al otro y la otra, cómo nos nombramos en esa relación y cómo nombramos la propia relación en estos espacios. Lo anterior constituiría uno de los elementos centrales de lo que podría ser la base de una metodología dirigida hacia lo decolonial.

Esta última perspectiva de la transformación social dirigida hacia lo decolonial se encuentra en los testimonios de personas educadoras que se enuncian desde una (des)situación en la que comparten sus subjetividades y reflexiones críticas sobre su propia práctica. Así, las subjetividades y reflexiones de las personas-sujeto que en un movimiento de (des)situación se cuestionan su propia práctica y aluden a otras formas posibles de ejercerla representan un ejercicio de resignificación y, por tanto, una aproximación hacia lo decolonial en el encuentro pedagógico. De ahí que la situacionalidad y, sobre todo, la (des)situación se vuelven elementos importantes para pensar y reflexionar estos encuentros pedagógicos.

La situacionalidad sobre la que se asienta la práctica socioeducativa en algunos momentos en Amalgama sucede desde una práctica de intervención y depósito, lo cual implica tensiones y reproducción de elementos de la estructura colonial-capitalista-patriarcal, como la colonialidad del ser, saber y poder como lógicas sutiles de exclusión. No obstante, esta situacionalidad no es determinante, ya que también advertimos la posibilidad de la (des)situación de esta práctica de intervención en la reflexión crítica de las personas educadoras. Por ello, esta investigación invita a considerar que la práctica socioeducativa que reproduce algunas lógicas de exclusión en Amalgama, no es definitiva, sino que podría ser subvertida si se atiende la (des)situación de las personas-sujeto educadoras que revela las subjetividades y reflexiones críticas en un ejercicio que se aproxima a lo decolonial.

\section{REFERENCIAS BIBLIOGRÁFICAS}

Arroyo Ortega, A., García Labrador, J., Di Caudo, M. V., Ossola, M. M., Mancinelli, G., Hecht, A. y Otaso, A. (2016). Interculturalidad y educación desde el sur: contextos, experiencias y voces. Recuperado de http://biblioteca.clacso.edu.ar/clacso/ se/20161004101819/Interculturalidad_y_educacion.pdf

Asamblea General de las Naciones Unidas (1948, diciembre). Declaración de los Derechos Humanos. Resolución 2017 A (III).

Bárcena, F., Larrosa, J. y Mélich, J. C. (2006). Pensar la educación desde la experiencia. Revista Portuguesa de Pedagogía, vol. 40, núm. 1, pp. 233-259. 
Bauman, Z. (2003). La comunidad, en busca de la seguridad en un mundo hostil. España: Siglo XXI.

Berlanga, B. (2014a). Educar con sujeto: experiencia, don y promesa (otro que no sea el de la intervención). Puebla, México: UCI-RED CESDER. Recuperado de https:// docs.google.com/file/d/OB2GOSriU9VAwemVQRXhMZkx6ZXM/view

Berlanga, B. (2014b). Fragmentos acerca del artilugio en la pedagogía del sujeto (pp. 1-9). Puebla: UCI-RED CESDER. Recuperado de https://docs.google.com/a/ecosur. edu.mx/file/d/OB2G0SriU9VAwekM1MC1fVW1aTmc/view

Bertely-Busquets, M. (2016). Políticas neoliberales y afectaciones territoriales en México. Algunos "para qués" de "otras" educaciones. LiminaR. Estudios Sociales y Humanísticos, vol. XIV, núm. 1, pp. 30-46. Recuperado de http://liminar.cesmeca. $\mathrm{mx} /$ index.php/r1/article/view/421

Camacho, H., Cámara, L., Cascante, R. y Sainz, H. (2001). El enfoque del marco lógico: 10 casos prácticos. Cuaderno para la identificación y diseño de proyectos para el desarrollo. Madrid, España: CIDEAL.

Coffey, A. y Atkinson, P. (2003). Encontrar el sentido a los datos cualitativos: estrategias complementarias de investigación. Medellín, Colombia: Editorial Universidad de Antioquia.

Colmenares, E. (2012). Investigación-acción participativa: una metodología integradora del conocimiento y la acción. Voces y Silencios. Revista Latinoamericana de Educación, vol. 3, núm. 1, pp. 102-115.

Comboni, S. S. y Juárez, N. J. M. (2013). Las interculturalidad-es, identidad-es y el diálogo de saberes. Reencuentro, núm. 66, pp. 10-23.

Del Rincón, D., Arnal, J., Latorre, A. y Sans, A.(1995). Técnicas de investigación en ciencias sociales. Madrid, España: Dykinson.

De Sousa, B. (2009). Más allá del pensamiento abismal. De las líneas globales a una ecología de saberes. Para descolonizar el Occidente más allá del pensamiento abismal (pp. 11-44). En L. Tapia Mealla (coord). Pluralismo epistemológico. La Paz: Cide Umsa.

De Sousa, B. (2003). Crítica de la razón indolente. Contra el desperdicio de la experiencia. Para un nuevo sentido común: la ciencia, el derecho y la política en la transición paradigmática. Bilbao, España: Desclée De Brouwer, SA.

Dietz, G., Selene, L. y Mateos, C. (2002). Universidades interculturales en México. Revista de Investigación Educativa, núm. 21, pp. 683-690.

Dussel, E. (1993). Eurocentrism and modernity (Introduction to the Frankfurt Lectures). Boundary, vol. 20, núm. 3, pp. 65-76.

Estermann, J. (2014). Colonialidad, descolonización e interculturalidad: apuntes desde la filosofía intercultural. Polis (Santiago), vol. 13, núm. 38, pp. 347-368.

Foucault, M. (1988). El sujeto y el poder. Revista Mexicana de Sociología, vol. 50, núm. 3, pp. 3-20.

Freire, P. (1967). La educación como práctica de la libertad. Caracas, Venezuela: Nuevo Orden.

García Aguilar, M. del C. (1998). Las organizaciones no gubernamentales en Chiapas: algunas reflexiones en torno a su actuación política. En Anuario 1997 del Centro de Estudios Superiores de México y Centroamérica de la Universidad de Ciencias y Artes del Estado de Chiapas (pp. 37-80). Universidad de Ciencias y Artes de Chiapas, Centro de Estudios Superiores de México y Centroamérica. 
Gasché, J. (2008). La motivación política de la educación intercultural indígena y sus exigencias pedagógicas. ¿Hasta dónde abarca la interculturalidad? Educando en la diversidad. Investigaciones y experiencias educativas interculturales y bilingües (pp. 367-397). Quito, Ecuador: Ediciones Abya-Yala

Giner, A., Saldívar, A., Duarte, J. y Keck, C. (2017). Interculturalidad crítica y educación no formal: reflexiones desde Amalgama, AC, en San Cristóbal de Las Casas, Chiapas, México (tesis). El Colegio de la Frontera Sur, San Cristóbal de Las Casas, México.

González, G. (2002). Sociedad civil, organismos civiles y movimientos populares en los Altos y la Selva de Chiapas. Ciudad de México: Universidad Autónoma Metropolitana, Unidad de Xochimilco.

Grosfoguel, R. (2013). Racismo/sexismo epistémico, universidades occidentalizadas y los cuatro genocidios/epistemicidios del largo siglo XVI. Tabula Rasa, núm. 19, pp. 31-58.

Gunther, D. y Cortés, L. (2011). Interculturalidad y educación intercultural en México: un análisis de los discursos nacionales e internacionales en su impacto en los modelos educativos. México, DF: Secretaría de Educación Pública.

Gutiérrez, R. (2006). Impactos del zapatismo en la escuela: análisis dinémica educativa indígena en Chiapas (1994-2004). LiminaR. Estudios Sociales y Humanísticos, vol. 1, núm. 1, p. 21. Recuperado de http://doi.org/http://dx.doi.org/10.2536/ liminar.v4i1.199

Harding, S. (1996). Ciencia y feminismo. Madrid: Ediciones Morata.

Lander, E. (1993). La colonialidad del saber: eurocentrismo y ciencias sociales. En Perspectivas latinoamericanas. Caracas: Facultad de Ciencias Económicas y Sociales, Instituto Internacional de la Unesco para la Educación Superior en América Latina y el Caribe.

Larrosa, J. (2003). Experiencia y pasión. Entre las lenguas. En Lenguaje y educación después de Babel (pp. 165-178). Barcelona, España: Editorial Laertes.

Lugones, M. (2011). Hacia un feminismo descolonial. La Manzana la Discordia, vol. 6, núm. 2, pp. 105-117. Recuperado en http://revistas.univalle.edu.co/index.php/ la_manzana_de_la_discordia/article/view/1504

Maldonado, N. (2003). Sobre la colonialidad del ser: contribuciones al desarrollo de un concepto. En Santiago Castro-Gómez y Ramón Grosfoguel (eds.). El giro decolonial. Reflexiones para una diversidad epistémico más allá del capitalismo global (pp. 127-168). Bogotá: Siglo del Hombre.

Martín, R. B. (2013). Contextos de aprendizaje: formales, no formales e informales. Ikastorratza e-Revista de Didáctica, núm. 12, pp. 1-14. Recuperado de http:// www.ehu.eus/ikastorratza/12_alea/contextos.pdf

Mélich, J. C. (2008). Antropología narrativa y educación. Teoría de la Educación. Revista Interuniversitaria, núm. 20, pp. 101-124.

Mignolo, W. (2005). El pensamiento des-colonial, desprendimiento y apertura: un manifiesto. Coimbra: Universidad de Coimbra.

Peces-Barba, G. (2002). La universalidad de los derechos humanos. Revista Doxa, vol. 15, núm. 2, pp. 613-633. Recuperado de http://www.corteidh.or.cr/tablas/ r28867.pdf

Pérez Luño, A. E. (1998). La universalidad de la declaración de las Naciones Unidas. Persona y Derecho, núm. 38, pp. 11-34. Recuperado de http://www.attac. es/2012/03/18/los-deberes-humanos/ 
Pincheira Muñoz, L. (2013). Radio comunitaria, un espacio educativo no formal en la comunidad. Rexe. Recuperado de http://www.rexe.cl/ojournal/index.php/rexe/ article/view/73/68

Quijano, A. (2002). Colonialidad del poder, eurocentrismo y América Latina. En Edgardo Lander (comp.). La colonialidad del saber: eurocentrismo y ciencias sociales. Perspectivas latinoamericanas. Buenos Aires, Argentina: CLACSO.

Rivas, J. (2005). Pedagogía de la dignidad de estar siendo. Entrevista con Hugo Zemelman y Estela Quintar. Revista Interamericana de Educación de Adultos, vol. 27, núm. 1, pp. 113-140.

Rubin, G. (1986). El tráfico de mujeres: notas sobre la "economía política del sexo". Nueva Antropología, vol. VIII, núm. 30, pp. 95-145. Recuperado de http://www. redalyc.org/pdf/159/15903007.pdf

Sartorello, S. C. (2009). Perspectiva crítica sobre interculturalidad y educación intercultural bilingüe: el caso de la unión de maestros de la nueva educación para México (UNEM) y educadores independientes en Chiapas. Revista Latinoamericana de Educación Inclusiva, vol. 3, núm. 2, pp. 77-90. Recuperado de http://www.rinace.net/rlei/numeros/vol3-num2/art5.html.

Schmelkes, S. (2003). Creación y desarrollo inicial de las universidades interculturales en México: problemas, oportunidades. retos. Diversidad cultural e interculturalidad en educación superior. En Experiencias en América Latina (pp. 329-339). Caracas:IESALC-Unesco.

Smitter, Y. (2006). Hacia una perspectiva sistémica de la educación no formal. Laurus, vol. 12, núm. 22, pp. 241-256 [revista impresa]. Recuperado de http://www.redalyc.org/articulo.oa?id=76102213C

Taylor, S. J. y Bogdan, R. (1987). Introducción a los métodos cualitativos de investigación. La búsqueda de significados. Buenos Aires, Argentina: Paidós.

Turbino, F. (2004). Del interculturalismo funcional al interculturalismo crítico. Rostros y Fronteras de la Identidad, núm. 151, pp. 1-9.

Trilla, J. (1993). Otras educaciones. Barcelona, España: Anthropos.

Uribe, G. y Martínez, J. (2012). Cambio religioso, expulsiones indígenas y conformación de organizaciones evangélicas en Los Altos de Chiapas. Política y Cultura, núm. 38, pp. 141-161.

Viñao, A. (2012). Higiene, salud y educación en su perspectiva histórica. Areas. Revista Internacional de Ciencias Sociales, núm. 20, pp. 9-24.

Walsh, C. (2017). Gritos, grietas y siembras de vida: entretejeres de lo pedagógico y lo decolonial. En Pedagogías decoloniales. Prácticas insurgentes de resistir, (re) existir y (re) vivir, tomo II (pp. 17-48). Quito: Editorial Abya Yala. Recuperado de https://moarquech.files.wordpress.com/2017/08/ferrera_pedagogias_decoloniales_tomoii_libro.pdf

Walsh, C. (2010). Interculturalidad crítica y educación intercultural. En Construyendo la interculturalidad crítica (pp. 75-96). Bolivia: Instituto Internacional de Integración del Convenio Andrés Bello. Recuperado de http://aulaintercultural. org/2010/12/14/interculturalidad-critica-y-educacion-intercultural

Walsh, C. (2009a). Interculturalidad crítica y pedagogía de-colonial: apuestas (des) de el in-surgir, re-existir y re-vivir. UMSA Revista (entre palabras), núm. 3, pp. 3031. Recuperado de https://redinterculturalidad.files.wordpress.com/2014/02/ interculturalidad-crc3adtica-y-pedagogc3ada-decolonial-walsh.pdf 
Walsh, C. (2009b). Interculturalidad, estado, sociedad: luchas (de)coloniales de nuestra época. Quito: Universidad Andina Simón Bolívar. Recuperado de http://www. flacsoandes.edu.ec/interculturalidad/wp-content/uploads/2012/01/Interculturalidad-estado-y-sociedad.pdf

Walsh, C. (2009c). Interculturalidad, colonialidad y educación. (Etno)educación, Multiculticulturalidad e Interculturalidad, núm. 12. Recuperado de http://www.flacsoandes.edu.ec/sites/default/files/agora/files/1265909654.interculturalidad_ colonialidad_y_educacion_0.pdf

Walsh, C. (2005). Interculturalidad, conocimientos y decolonialidad. Signo y Pensamiento, vol. XXIV, núm. 46, pp. 39-50. Recuperado de https://www.researchgate. net/publication/237041504_Interculturalidad_conocimientos_y_decolonialidad

Zemelman, H. (2012). Pensar y poder. Razonar y gramática del pensar histórico. México: Siglo XXI/Universidad de Ciencias y Artes de Chiapas. Recuperado de https:// dlscrib.com/download/pensar-y-poder-razonar-ygram-aacute-ticadel-pensarhist-oacute-rico-hugozemelman_58d41e3edc0d600234c34645_pdf

Zemelman, H. (2010). Sujeto y subjetividad: la problemática de las alternativas como construcción posible. Polis, núm. 9, PP. 355-366. Recuperado de http://journals. openedition.org/polis/943 